\title{
Research on Digital Twin and Collaborative Cloud and Edge Computing Applied in Operations and Maintenance in Wind Turbines of Wind Power Farm
}

\author{
Fuxing $\mathrm{LI}^{\mathrm{a}, 1}$, Luxi LI ${ }^{\mathrm{b}}$ and You PENG ${ }^{\mathrm{b}}$ \\ a Academic Affairs Office, Southeast University, Nanjing, China \\ ${ }^{\mathrm{b}}$ School of Trade and Logistics, Jiangsu Vocational and Technical College of \\ Economics and Trade, Nanjing, China
}

\begin{abstract}
For the increasingly prominent problems of wind turbine maintenance, using edge cloud collaboration technology to construct wind farm equipment operation and maintenance framework is proposed, digital twin is used for fault prediction and diagnosis. Framework consists of data source layer, edge computing node layer, public or private cloud. Data source layer solves acquisition and transmission of wind turbine operation and maintenance data, edge computing node layer is responsible for on-site data cloud computing, storage and data transmission to cloud computing layer, receiving cloud computing results, device driving and control. The cloud computing layer completes the big data calculation and storage from wind farm, except that, based on real-time data records, continuous simulation and optimization, correct failure prediction mode, expert database and its prediction software, and edge node interaction and shared intelligence. The research explains that wind turbine uses digital twin to do fault prediction and diagnosis model, condition assessment, feature analysis and diagnosis, life prediction, combining with the probabilistic digital twin model to make the maintenance plan and decision-making method.
\end{abstract}

Keywords. Digital twin, edge-cloud collaboration, wind turbine, equipment operation and maintenance, prognostics; remaining useful life

\section{Introduction}

At present, the operation and maintenance of wind turbines (wind turbines for short) lags behind the development of the wind power industry. Most of them adopt manual operation and maintenance mode. Wind power equipment maintenance involves many parts and links, and the manufacturer's own equipment detection system has compatibility problems. Wind turbines are usually installed in remote locations, far apart, and inconvenient transportation, which causes a lot of inconvenience to the maintenance of wind power equipment. The equipment is installed at the end of a wind power tower with a height of tens or even hundreds of meters, which is difficult to maintain and costly. Statistics show that maintenance costs for onshore and offshore

1 Corresponding Author, Fuxing LI, Academic Affairs Office, Southeast University, Nanjing, China; Email: 1253059637@qq.com. 
wind turbines account for $10 \%$ to $15 \%$ and $20 \%$ to $35 \%$ of wind farm revenue [1]. In order to improve the operating efficiency of wind turbines and reduce maintenance costs [2], scholars have done a lot of research on the failure prediction and diagnosis modes, prediction algorithms, and diagnosis mechanisms of the main components of a single set of wind turbines [3]. Research on the operation and maintenance of wind turbines in the entire wind farm. This paper proposes to use edge-cloud collaboration technology to build a full-field wind turbine operation and maintenance platform to achieve unified real-time measurement and control of wind turbine operation and maintenance. The edge computing end is responsible for the daily operation and maintenance data processing of the wind turbine on-site, and cloud computing is responsible for the real-time data of the entire wind farm. Recording, visualization, data analysis, optimization and correction of fault prediction and diagnosis modes and algorithms, and downloading the results to edge computing nodes. Aiming at the fault operation and maintenance prediction of a single set of wind turbines, it is proposed to adopt digital twin and probabilistic twin digital drive technology to realize fault prediction and diagnosis.

\section{Wind Turbine Fault and Maintenance Status}

Common electrical fault operation and maintenance costs of wind turbines account for about $70 \%$ of the entire wind turbine operation and maintenance costs, and other parts account for about $30 \%$. The approximate distribution rate of the faults of each component of the wind turbine is shown in figure 1 [4], and the approximate time required for shutdown and maintenance caused by the failure of each component is shown in figure 2 [4].

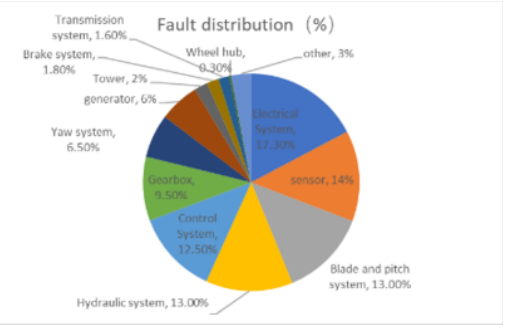

Figure 1. Proportion of failure of each component.

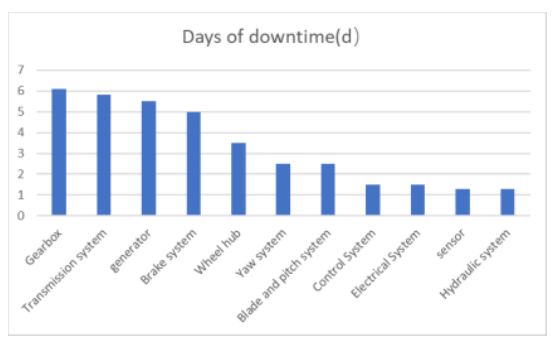

Figure 2. Downtime due to component failure.

The parts that frequently fail are generators, main shafts, gearboxes, yaw systems, pitch systems, hydraulic systems, racks and wind turbine control systems. For example, wind power mainly relies on the rotation of the turbine blades to convert kinetic energy into electrical energy, and the movement speed of the blade tip reaches about $300 \mathrm{~km} / \mathrm{h}$. Long-term work in the field under the sun and rain will cause corrosion, cracks or degradation of the blade coating, which will affect its performance and then affect the power generation. At present, there is no good way to detect the blade health status, and there is a lack of blade maintenance historical data. Statistics show that the faults of wind turbines caused by sensors account for about $14 \%$, and the faults caused by sensor-related system damage account for about $40 \%$ [5]. 


\section{Edge-Cloud Collaborative Wind Turbine Operation and Maintenance Platform}

The platform refers to version 3.0 of the edge computing reference architecture proposed by the Edge Computing Alliance, which is composed of a terminal data source layer, an edge computing node layer, and a public cloud/private cloud layer, as shown in figure 3 . The edge computing node layer is between the public cloud/private cloud layer and the terminal data source layer. One end receives data sent by various devices at the terminal data source layer and controls the equipment, and the other end performs service collaboration and business management collaboration with the public cloud and private cloud layer. Application management collaboration, intelligent collaboration, data collaboration and resource collaboration.

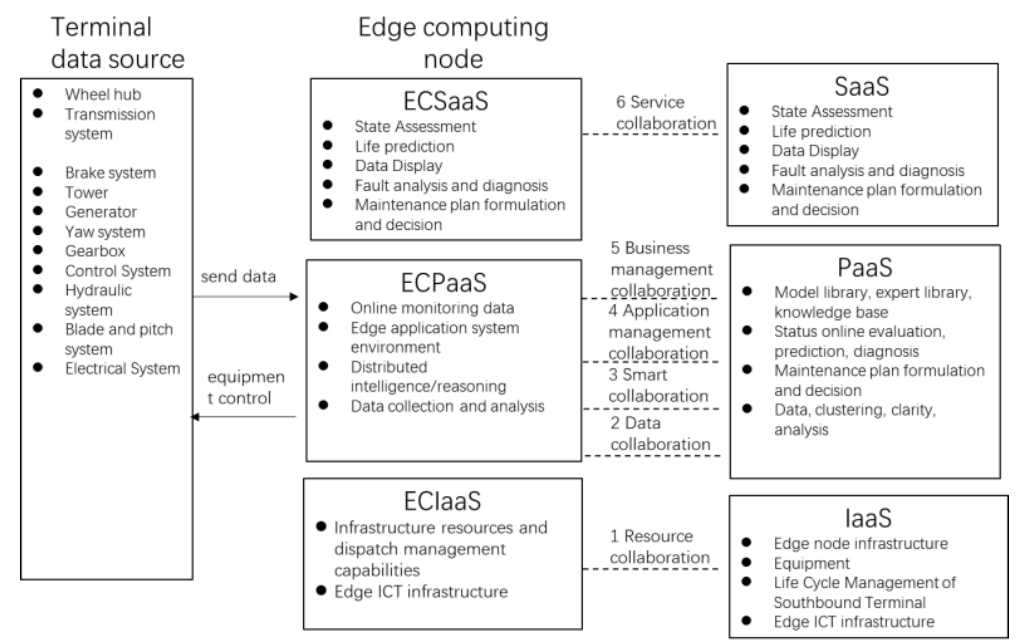

Figure 3. Wind turbine operation and maintenance framework.

\subsection{Platform Terminal Data Source Layer}

The terminal data source layer is composed of various data interfaces of wind turbine equipment, parts and instruments. The large amount of data generated by the operation of these equipment is the basis of digital operation and maintenance. If these devices need to implement digital operation and maintenance but do not have a data output interface, wired or wireless shock and vibration sensors or acceleration sensors can be installed at the predicted location of the device to form a sensor network, which can be collected and transmitted in real time. Data to intelligent edge devices. At present, various sensors installed in wind turbines can transmit data with data terminals through RS-232, RS-485, input and output interfaces, and use MODBUS protocol to connect to various popular network interfaces, network protocols, network topologies, Internet deployment and configuration, system management and protection, etc., can make full use of and absorb advanced technologies in the Internet field and various industrial bus interconnections. Wind turbines are distributed in a wide area. Edge computing is the edge computing of the network platform that is close to the object or data generating end. The terminal data source layer completes sending data to the edge node computing layer and accepts the control of the edge computing node. 


\subsection{Platform Edge Computing Node Layer}

The edge computing node layer is composed of the infrastructure layer (ECIaaS), the edge computing platform layer (ECPaaS) and the service (ECSaaS) layer. The ECIaaS layer is composed of edge gateways, edge controllers, edge clouds, edge sensors and other hardware, with control function modules, analysis function modules, optimization function modules, computing network storage calling interfaces, various computing capabilities, network resources, storage resources, etc., It is the business core based on the digital twin and edge-cloud collaborative system. Edge nodes are responsible for high-reliability data collection and processing, and receive equipment failure prediction and diagnosis models established or iterated by the back-end cloud computing platform based on big data calculations, expert libraries and rules, product knowledge bases, etc., and realize equipment on-site early warning.

The ECPaaS layer is responsible for edge device data collection and analysis, distributed intelligence/reasoning, edge application environment, online monitoring, etc., and the data source layer data reception and control instructions, and the cloud computing platform for data, intelligence, application management, and business of synergy. Data collection enters the edge layer through fieldbus, device network, wireless network, mobile communication, etc. Distributed intelligence/reasoning is completed by edge computing resources, which are composed of the CPU, memory, and read-write interface resources of each edge intelligent node device. When the computing power of a single edge node is limited, edge computing can call the CPU and memory of other edge nodes according to the rules to meet the needs at the time, and unload the task after the calculation is completed to release the CPU and memory resources. The storage resource is responsible for storing the data collected, generated and received by the edge device in the memory and disk storage of the edge device in accordance with the distributed storage rules. Data reception and collaboration are completed through network resources. Network resources mainly involve mobile communications, broadband Internet, Internet of Things, multi-source shared databases, etc., responsible for data upload and release, upload and upload useful data to the cloud computing platform, and release is cloud computing. The results and control instructions are transmitted to the equipment at the data source layer. In addition, calling API module, control module, analysis module and optimization are all functional modules that support optimization of fault prediction and diagnosis.

The ECSaaS layer consists of edge computing application software, such as online monitoring and evaluation, equipment failure analysis and diagnosis, remaining life prediction, failure prediction, maintenance plan formulation and decision-making, etc., and performs service application-level collaboration with cloud computing.

\subsection{Platform Public Cloud/Private Cloud Layer}

The cloud computing layer is composed of a large number of servers, storage devices, network devices, and system display devices that constitute the backend of the entire wind farm equipment management. It links with edge computing node equipment through the Internet to complete system big data processing and send instructions to the edge layer, so that edge computing nodes can complete software upgrades, model corrections, algorithm iterations, etc. in time. At the same time, the received big data is displayed, and if the system fails, an early warning will be sent in time. Workers obtain real-time production information, equipment operating conditions, abnormal alarms, 
production scheduling decisions, etc. through the terminal, and issue instructions to edge computing nodes through the terminal device. This layer uses data mining algorithms and artificial intelligence algorithms to clean, cluster, and mine the operating data of these equipment based on the huge amount of data collected by similar wind turbine equipment, and establish or iterate fault diagnosis expert knowledge. Ref. [6], constantly updates the expert knowledge base and constructs new fault diagnosis rules. The expert knowledge base and diagnosis rules can be downloaded to the intelligent edge terminal, improving the speed of equipment diagnosis.

\section{Device Operation and Maintenance Driven by Digital Twin (DT)}

\subsection{Overview of Key Technologies for the Operation and Maintenance of DT Drive Equipment}

The concept of DT was put forward by Grieves [7] in the product life cycle management course of the University of Michigan in 2003. Ref. [8] defines a DT as: a technical means with real-time synchronization and original mirroring, which can realize the interaction and integration of the physical world and the digital world. In 2017, researches on DT modeling, physical information fusion and service application have been carried out, focusing on the analysis of DT technology and related industry relations, the establishment of virtual models, the use of twin data fusion analysis, service application guidelines, etc. [9]. The essence of the DT is to use digital technology to complete real-time data recording, real-time simulation optimization, and virtual verification of physical entities, so that physical entities can be simulated, monitored, controlled, fault diagnosed, predicted, and early-warned. The DT drive system is mainly composed of three parts: a physical entity, a physical multi-dimensional model, and a data channel composed of sensors and other devices. The data channel realizes the interaction and common intelligence between the physical entity and the physical multi-dimensional digital model. The architecture diagram is shown in figure 4 shown.

The implementation roadmap for fault prediction and maintenance using digital twin technology includes the creation of DT; acquisition of multi-dimensional heterogeneous data; preprocessing of multi-dimensional heterogeneous data; model training and creation of equipment failure prediction and diagnosis models or algorithms; prediction of equipment failures based on algorithms and diagnosis of faults; six steps of equipment operation and maintenance for predicting equipment life and maintenance plan formulation and decision-making (figure 5). 


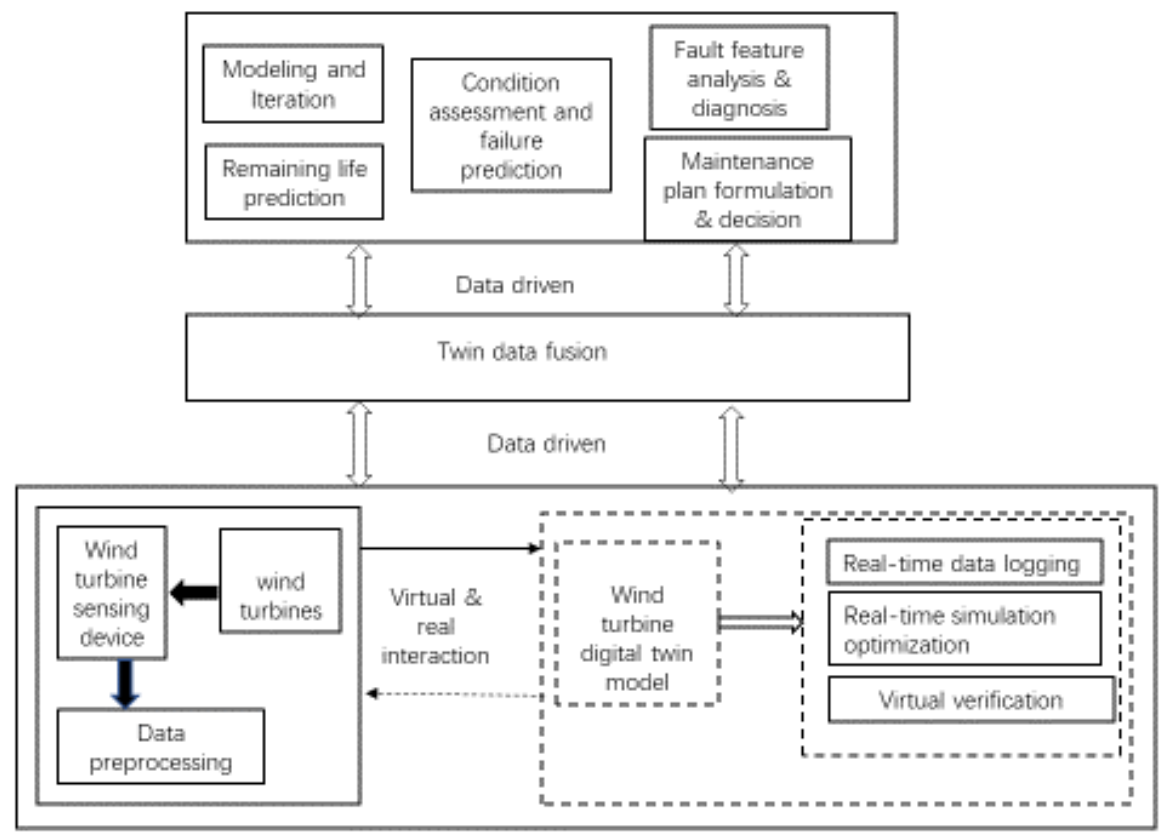

Figure 4. Based on the DT model.

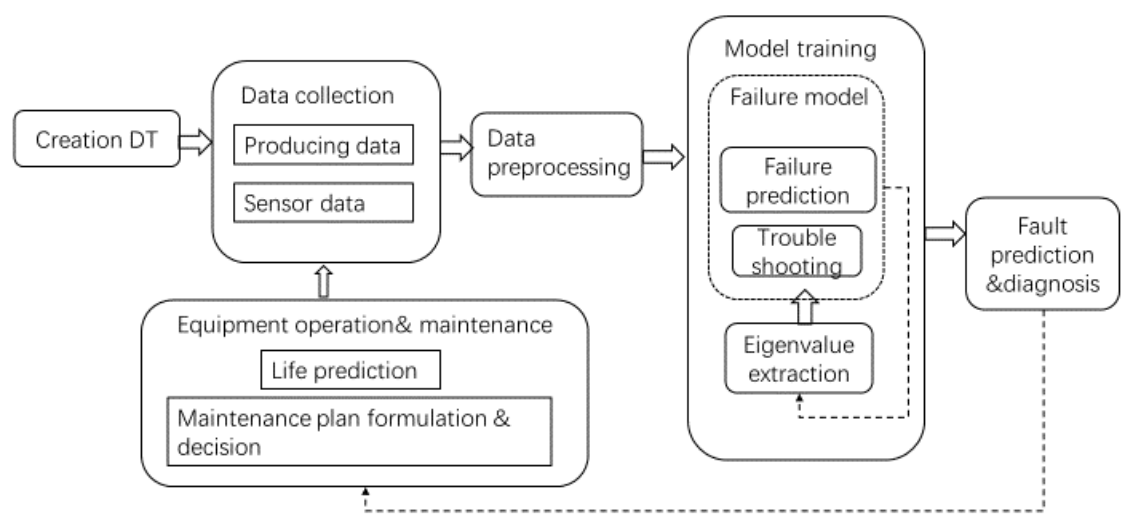

Figure 5. Steps of DT drive equipment operation and maintenance.

\subsection{Step 1: Creation of the Digital Twin}

The step1 is the creation of a digital twin. DT modeling focuses on equipment physical performance characteristics and equipment operating state parameters. Model parameters should be consistent with physical equipment operating data. The key to digitizing physical entities is the extraction of physical entity-related parameters and the establishment of prediction models. The extraction of physical entity parameters can first decompose the functions of the physical entities, and then digitize the functions to establish a DT system and physical device models. However, when 
extracting parameters, multiple iterations are required to establish a complete virtual-real consistency model. Modeling can also use mature software, for example: MathWorks' MATLAB, Simulink, Simscape and other software packages. The CAD drawings and 3D models of the equipment used to create the DT can generally be obtained from the original factory. After importing the equipment plane or 3D drawing into some commercial software, a multi-body interactive simulation model of the equipment running 3D model can be constructed. The construction of the equipment digital twin model requires high calculation and memory requirements for the system. Therefore, the modeling process can be carried out on the cloud computing platform. By using the cloud computing platform's strong computing power and large storage space, a variety of modeling software can be installed to adapt to the need to model parts with different characteristics. After the model is built, it is installed on the edge node to realize on-site inspection and evaluation.

\subsection{Step 2: Obtain Multi-dimensional Heterogeneous Data}

The step2 is to obtain multi-dimensional and heterogeneous data. The operation and maintenance of equipment is mainly to judge the operating status of the equipment based on the multi-dimensional heterogeneous data such as equipment working condition data and numerous sensor data on site. These data are divided into two categories, one is the data in the normal state of the device, and the other is the data in the abnormal state of the device.

The data to obtain the normal status of the equipment can be obtained from the real-time record of the equipment's daily operation status detection system (SCADA). Although these data may be insufficient and incomplete, the data can be increased by changing the system settings or adding detection points.

It is difficult to obtain data when the device is in an abnormal state. Under normal circumstances, there is very little data when the device fails. If the equipment is maintained too frequently in the field, there may be no faults and no fault data, or the equipment will never be allowed to fail, and there will be no fault data. To this end, data can be obtained from other departments within the enterprise, or from partners upstream and downstream of the supply chain. In order to prevent the lack of failure data from becoming a fatal obstacle to predictive maintenance, simulation tools can also be used to generate test data and combine this data with sensor data that can be used to construct and verify predictive maintenance algorithms, such as Simulink from MathWorks, etc. The tool simulates fault data, recognizes warning signals from available operating data, and generates sample failure data from the simulation tool; it can also dig out fault data from the trend of equipment operating efficiency decline, and use statistical methods such as PCA to reduce dimensionality to discover massive process data the rule of summarization, and the resulting fault data is used as equipment operation and maintenance data.

\subsection{Step 3: Multi-dimensional Heterogeneous Data Preprocessing}

In the equipment operation and maintenance system driven by the digital twin, the physical entity data is also input into the digital twin at the same time, so that the digital twin and the physical entity operate synchronously. These data will enter the digital twin after data cleaning, integration and transformation, and data reduction, and be used for the creation of fault prediction and diagnosis models. 
Data cleaning. In the equipment operation and maintenance system driven by the digital twin, the automatically collected data may be missing, and there are multiple algorithms for filling the missing data. Considering the large amount of real-time detection data of the equipment, the mean filling method is used to fill in the occasional missing data. The method of missing data has little effect on the accuracy of the data.

Data integration and transformation. Data integration is to merge data from multiple data sources and store them in a consistent data store (such as a data warehouse). These data sources may include multiple databases, data cubes or general files. Data integration has three main problems: eliminate redundancy Monitoring and processing of residual data and data value conflicts and pattern integration and object matching. Data transformation is to transform or unify the data into a form suitable for mining. The method is very poor normalization transformation, feature scaling and so on. There are three types of range normalization transformation: minimum-maximum normalization, z-score normalization, and decimal calibration normalization. The minimum-maximum normalization method is selected in the preprocessing of wind turbine operation and maintenance data.

Min-max normalization is a linear transformation of the original data, mapping the data value to $[0,1]$. The conversion formula is as follows:

$$
\mathrm{x}^{*}=(\mathrm{x}-\mathrm{min}) /(\max -\min )
$$

In equation (1), $x^{*}$ is the normalized data, $x$ is the original data, and min and max are the smallest and largest data in the data, respectively. This method preserves the relationship between the original data and is also the simplest method. The disadvantage is that if the values are concentrated and a certain value is large, each value after normalization is close to 0 , and there will be little difference. (Such as 1.1, $1.2,1.3,1.4,1.5,1.6,9.4$ this set of data). If the value range of the current attribute [min, max] is exceeded in the future, the system will report an error, and the min and max need to be re-determined. In order to solve this problem, a sliding window is opened for the data source according to the time sequence, and the window data is continuously extracted for normalization, so as to obtain a batch of more accurate normalized data, and then use the min-max method to iterate to obtain relatively accurate data.

Data reduction. Data reduction is to obtain a data subset whose result is much smaller than the original data source, but still close to maintaining the integrity of the original data, which is convenient for calculation and processing. For the scene of digital twin application collecting big data on-site, there are two types of reduction for multi-source heterogeneous data sources: quantity reduction and dimensionality reduction. The cluster analysis method of quantity reduction uses the cluster center data as a subset of the original data; the dimensionality reduction extracts the data of the main dimensions from the original data, so as to reduce the number of variables or attributes in the data and map the original data to in a space with a small dimension, the data corresponding to the dimension with a large amount of information is retained, and the dimensionality reduction is divided into lossless and lossy. For the digital twin driving mode, the dimensionality reduction adopts a hybrid method of wavelet transform and principal component analysis. The methods and algorithms of multi-dimensional heterogeneous data preprocessing are not only the above, they need to be further verified in practice. 


\subsection{Step 4: Model Training}

Model training includes the creation of equipment failure prediction and diagnosis models or algorithms, and feature value extraction. The digital twin driving mode is based on the establishment of physical digital twins and real-time online detection, using real-time dynamic data to create fault prediction models and algorithms. In order to obtain more equipment failure information, the digital twin has a dynamic interactive function and the failure information is added to the model. For example, adding short-circuit or open-circuit faults of electrical equipment lines, mechanical bearing wear, and changing parameter values can simulate the consequences of faults.

In order to simulate the dynamic behavior of the device, the digital twin can also add parts and parameters of commonly used electromechanical equipment. These parts and their parameters can be found in the general commercial software product library. If there is a lack of parameters, commercial software can also be used to automatically adjust these parameter values so that the parameters generated by the simulation model match the measured data of the physical entity. After the simulation of each function of the device is completed, the digital twin is "assembled" into a complete device to achieve the effect of fully matching the physical entity. The data obtained through simulation can be used as reference data for equipment operation, as well as reference data for realizing fault prediction and diagnosis, as well as characteristic values of fault prediction and diagnosis. In order to improve the effect, simulation scenarios and times can be added to the cloud computer layer. The more times, the closer the simulation effect is to reality.

The simulation results are used to extract training data for machine learning algorithm. With the development of machine learning, deep machine learning has been gradually promoted, and many algorithms to solve prediction problems have also been produced, such as: dynamic Bayesian network graph model, particle filter inference algorithm to estimate the state and parameters of equipment at the same time, and Gaussian particle filter, etc. Algorithm for reasoning verification [10] and other means of multi-parameters and models, prediction algorithms, artificial intelligence, etc. to realize the operation and interaction between physical entities and digital models to complete equipment failure prediction tasks, but different algorithms have different requirements for training data. For the fault prediction and diagnosis data, the training data is scarce, and the data needs to be extracted through simulation. In order to reduce the dependence of the prediction on the training data, the Markov model of deep learning is selected, which does not need to use too much prior knowledge. The deep learning method can be used to adaptively find the pattern characteristics between the input trend data and the trend data to improve the prediction accuracy. Because the system adopts a side-cloud collaborative framework, utilizes the computing and storage capabilities of cloud computing, and adopts different models and algorithms for different devices and components, effectively improving the accuracy of fault prediction. Through model training, equipment failure prediction and diagnosis characteristic values are obtained, which creates conditions for equipment failure prediction and diagnosis.

\subsection{Step 5: Equipment Failure Prediction and Diagnosis}

Equipment failure prediction and diagnosis can be based on a data-driven model. The common types of prediction algorithms in the data-driven model include supervised 
learning and unsupervised learning. Supervised learning methods include decision trees, random forests, support vector machines, traditional neural networks and deep learning; unsupervised learning methods include kernel density estimation, k-means clustering and principal component analysis. Samir K summarized these methods in Ref. [11] and pointed out that decision trees need to use past experience data and there is overfitting; although random forest has improved decision trees, it is faster than support vector machines in training speed; Bayesian networks require prior knowledge, and the process of learning unknown structures is complicated; although the naive Bayesian algorithm is simple and requires less training data, it needs to assume that the data attributes are independent of each other, it also has pre-probability, and has high requirements for the input data format. The result of the change based on the feature combination cannot be processed, etc. Chen [12] studied the application of deep learning in equipment failure prediction and diagnosis, and proposed that deep learning can discover more hidden knowledge in the process of feature extraction of hierarchical structure, and has strong adaptability.

Troubleshooting. The digital twin fault diagnosis mode can be carried out according to various operating parameters and fault diagnosis decision-making modes and algorithms of wind turbines. For example: it can be carried out based on electrical signals, and a successful diagnosis of motor faults has been carried out [13]; Kia et al. [14] proposed using the digital twin model to study the fault of the motor drive system including the gearbox. The correlation between the current signal and the gearbox failure was obtained and verified by data analysis. These analysis and calculations can be done in the edge node server, or in the background cloud computing platform. After the results are obtained, they are sent back to the digital simulation equipment for simulation verification and the simulation results are verified. If the fault is resolved smoothly, it means that the solution is feasible, and then pre-maintain the physical entity to ensure the normal operation of the physical entity. On the contrary, the simulation results are fed back to the knowledge base and re-analyzed to find the root cause of the failure. This can not only maintain the existing equipment but also provide reference data for equipment upgrades.

For example: blade fault feature analysis and diagnosis under the digital blade, the digital blade simulation operation data can be compared with the actual collected data, if there is a difference in the data, then the uploaded inspection data can be used. The cloud computing platform uses data mining algorithms (such as random forest, K-nearest neighbors, AdaBoost, Naive Bayes to diagnose blade icing faults [15]), analyze and judge the fault, evaluate the damage condition of the blade, and obtain the auxiliary report of the fan blade loss. This front-end collects image data and transmits it to the back-end analysis of the edge-cloud collaborative mode, which can eliminate the differences in the manual inspection process, make each data more accurate and traceable to each inspection. Constructing a complete database for wind turbine inspections, realizing fault feature analysis and diagnosis, and establishing a digital foundation for equipment failure and life prediction.

The equipment operation and maintenance based on the digital twin-driven and edge collaborative environment has strong edge node computing and data center computing capabilities. Therefore, for the fault prediction and diagnosis of complex electromechanical equipment such as wind turbines, the model or algorithm can be based on the equipment zero Component features and simulation results are constantly adjusted without being stuck to a certain algorithm. This is the advantage of edge cloud architecture for equipment operation and maintenance. 


\subsection{Step 6: Equipment Operation and Maintenance}

\subsubsection{Prediction of Remaining Life of Equipment}

Equipment remaining service life (RUL) prediction and maintenance plan formulation and decision-making. With the help of digital twin technology, a model can be developed to estimate the RUL of the device, and the model can perform the estimation based on the time evolution or statistical properties of the condition index value. For example: there is a model that fits the time evolution of the status indicator and predicts how long it will take before the status indicator crosses a certain threshold indicating a fault condition. The model compares the time evolution of the status indicator with the measured or simulated time series of the system from operation to failure. The model can calculate the time when the current equipment is most likely to fail. In order to solve the uncertainty of the prediction model, the probability distribution of the RUL of the tested equipment can be provided. After determining possible condition indicators, a model algorithm design for RUL prediction can be developed.

DT technology has good scalability. On the one hand, it can meet the equipment service life predictions provided by different equipment suppliers of wind farms. It can be accessed during the entire equipment service life. The historical data of all online equipment during use can be updated and saved synchronously to ensure that the data can be traced back to the equipment. It provides real-time data comparison for the normal operation of the equipment and discovers the hidden dangers of the equipment in time; on the other hand, the data accumulated by the equipment operation of different manufacturers can be used for reference when making life expectancy and be shared by all node enterprises in the chain.

\subsubsection{Maintenance Plan Formulation and Decision}

Driven by the digital twin, the system operates synchronously according to the virtual and real data input from the outside, and obtains equipment evaluation, fault prediction and maintenance verification data. The purpose of the probabilistic digital twin model is to incorporate decision-making risk analysis, and will add a layer of probabilistic digital twin driving model to the existing digital twin model, as shown in figure 6 .

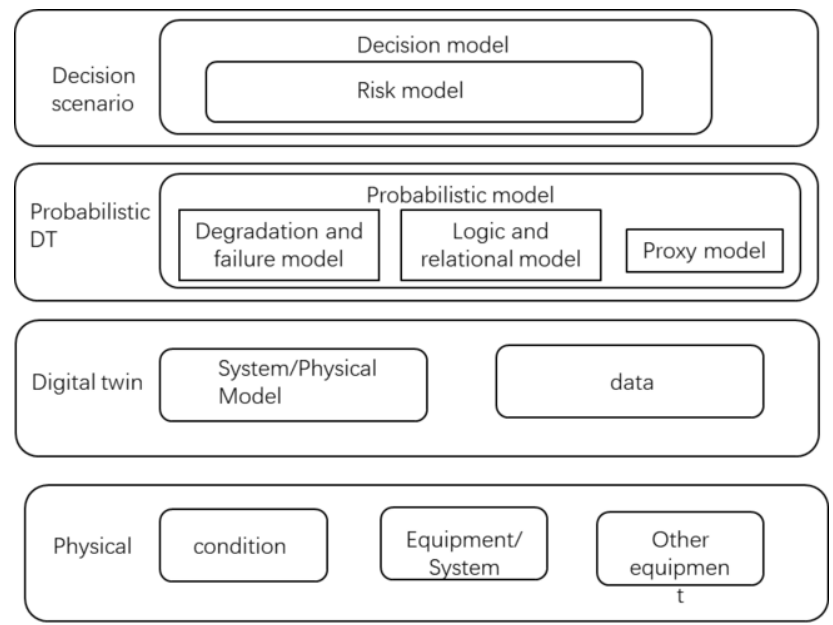

Figure 6. Probabilistic digital twin. 
The probabilistic digital twin drive model is divided into four layers. The first layer is the physical world, namely the equipment operating environment, physical equipment or systems, auxiliary equipment, etc. In the operation and maintenance of the wind turbine, in addition to some equipment on the wind turbine site, it should also include Computers, network equipment, sensor equipment, node servers, etc. The second layer is the digital twin layer, which mainly includes the system/physical digitized model system, data interface equipment, system display equipment, etc. The third layer is the probabilistic model of probabilistic digital twins, which mainly include equipment or system degradation and failure models, logic and relationship models, and proxy models. The fourth layer is the decision-making scenario layer, including various decision-making models, expert libraries, equipment procurement knowledge bases, risk analysis models, etc.

\section{Concluding Remarks}

Aiming at the difficulty in daily operation and maintenance of wind turbines in wind farms, a wind turbine operation and maintenance framework based on digital twins and edge clouds is proposed. The main components of the terminal data source layer, the edge computing node layer and the public cloud/private cloud that constitute the edge-cloud collaboration framework are described. It is proposed to use edge-cloud collaboration technology to build an operation and maintenance platform for wind turbines on site, and to unify real-time measurement and control of wind turbines. The edge computing end is responsible for the daily operation and maintenance data processing of wind turbines, and the background cloud computing is responsible for the data storage and data analysis of all wind farms, correcting fault prediction and diagnosis modes, and downloading the results to the edge computing node. The application of digital twin drive mode is proposed to predict and diagnose faults in the operation and maintenance of wind turbine equipment. It states the digital modeling method and iterative measures, failure prediction mode, failure feature analysis and diagnosis mode, life prediction mode and algorithm of wind turbines. The strategy of making equipment maintenance plan and decision based on probabilistic digital twin model is introduced. The application of digital twin technology has just started, and technologies such as equipment operation and maintenance modes, algorithms, and modeling methods driven by digital twins need to be further developed and improved.

\section{References}

[1] Fu Y, Yang F and Liu L 2019 Power System Technology 43 (11) 4057-63.

[2] Qiao W and Lu D G 2015 IEEE Transactions on Industrial Electronics 62 (10) 6536-45.

[3] Xiang J P and Jiang N N 2017 Journal of Chinese Society of Power Engineering 37 (10) 821-28.

[4] Ribrant J and Bertling L M 2007 IEEE Transactions on Energy Conversion 22 (1) 167-73.

[5] Jin X H, Qiao W and Peng Y Y 2016 IEEE Transactions on Industry Applications 52 (3) 2061-69.

[6] $\mathrm{Hu}$ W, Gao L and Fu L 2013 Chinese Journal of Scientific Instrument 34 (3) 524-30.

[7] Ren C and Chen L 2018 China Petroleum and Chemical Standard and Quality (21) 127-28.

[8] Tao F, Liu W R and Liu J H 2018 Computer Integrated Manufacturing Systems (1) 1-18.

[9] Wang J J, Xiang Y Q and He Z W 2019 Computer Integrated Manufacturing Systems 25 (6) 1348-60.

[10] Song Y, Shi Y Y and Yu J S 2019 Computer Integrated Manufacturing Systems 25 (6) 1559-66.

[11] Samir K and Takehisa Y 2018 Mechanical Systems and Signal Processing 107 241-65. 
[12] Chen Z Q, Chen X D and de Oliviral J V 2019 Chinese Journal of Scientific Instrument 40 (9) 206-21.

[13] Yacamini R, Smith K S and Ran L 1998 Journal of Vibration and Acoustics 120 (1) 72-79.

[14] Kia S H, Henal H and Capolino G A 2009 Proceeding of IEEE Energy Conversion Congress and Exposition 3182-87.

[15] Ye C L, Qiu Y G and Feng Y H 2018 Noise and Vibration Control 38 (z1) 643-47. 